\title{
Biomechanical assessment of reaching movements in post-stroke patients during a robot-aided rehabilitation
}

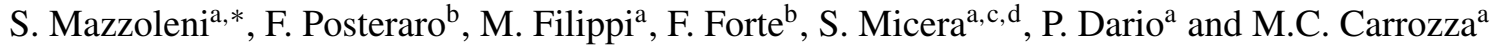 \\ ${ }^{\mathrm{a}}$ The BioRobotics Institute, Scuola Superiore Sant'Anna, Pisa, Italy \\ ${ }^{\mathrm{b}}$ Neurological Rehabilitation Unit, Auxilium Vitae Rehabilitation Center, Volterra, Italy \\ ${ }^{\mathrm{c}}$ Neuroprosthesis Control Group, Institute for Automation, Swiss Federal Institute of Technology \\ Zurich (ETHZ), Zurich, Switzerland \\ ${ }^{\mathrm{d}}$ Rehabilitation Institute and Technology Center Zurich, Zurich, Switzerland
}

\begin{abstract}
The main goal of this paper is to describe a method for the assessment of the motor performance in post-stroke subjects who have been undergone a robot-aided upper limb rehabilitation treatment.

The motivation for adopting such methodology relies on the need of quantitative methods for the evaluation of the effects of robot-aided rehabilitation treatments, which assumes great importance from the clinical point of view.

The method is based on the analysis of biomechanical parameters computed from force data recorded during the execution of planar reaching movements. Data from 17 chronic post-stroke patients and 5 healthy subjects were analysed.

The results show the effectiveness of the proposed method, which can contribute to quantitatively evaluate the effects of a robot-mediated therapy on the upper limb of chronic post-stroke subjects.
\end{abstract}

Keywords: Rehabilitation, robotics, post-stroke, upper limb, assessment

\section{Introduction}

After acute brain lesion, training has the potential to drive brain reorganization and to optimize functional performance $[4,28]$. Stroke and other neurological injuries cause partial destruction of the cortical tissue and result in a disturbed generation of motor programmes through the involvement of sensorimotor areas: a consequence is an impaired arm and hand motor function [29].

\footnotetext{
${ }^{*}$ Corresponding author: S. Mazzoleni, The BioRobotics Institute, Scuola Superiore Sant'Anna, Polo Sant'Anna Valdera, Viale R. Piaggio, 34 - 56025 Pontedera, Pisa, Italy. Tel.: +39 050883132; Fax: +39 050883101; E-mail: s.mazzoleni@sssup.it.
}

Therefore, optimal recovery of the upper limb function is essential for stroke patients to independently perform activities of daily living (ADL). Motor learning mechanisms are operative during the spontaneous stroke recovery and interact with rehabilitative training [13]. During the training, repetitive and novel tasks can be effective in reducing motor impairment after stroke [1].

Different robotic systems that can support movement therapy of the upper extremities in subjects with neurological pathologies were recently developed [26]. They can provide a safe and intensive motor therapy which can be highly accurate, intensive and prolonged. Recent systematic reviews showed that upper limb 
robot-aided rehabilitation treatments in post-stroke subjects improve short- and long-term motor control, even if no consistent influence on functional abilities was found [17, 23]. The studies which demonstrate the motor improvement are based on the analysis of the clinical scales scores: only few have proposed a biomechanical approach to analyse the mechanisms of motor recovery after stroke.

The comprehension of such mechanisms assumes great importance in the rehabilitation domain, as it can support the clinical decision process.

The question of whether the nervous system optimizes movements by a kinematic criterion, such as optimization of the trajectory error or smoothness, or a dynamic criterion, such as force or effort optimization, has been recently investigated [25]. Previous studies proposed a metric for assessing the forces exerted by post-stroke subjects during robot-aided rehabilitation treatment $[6,18,20]$.

The main goal of this study is to present a quantitative method based on biomechanical parameters for the assessment of the motor performance of post-stroke subjects who have been undergone a robot-aided upper limb rehabilitation treatment. The secondary goal is to demonstrate the effectiveness of such method, by demonstrating the hypothesis that the motor recovery of planar reaching movement in post-stroke patients after a robot-aided therapy is based on the preservation of biomechanical and physiological properties, instead of compensation mechanisms. For such purposes, preliminary results from an experimental group of 17 chronic post-stroke patients and 5 healthy subjects are presented.

\section{Methods}

\subsection{Subjects}

A group of 17 subjects, age range 33-66 (mean age $51.9 \pm 10.7$ ) years, thirteen men and four women, was recruited for the clinical trial (Table 1). Seven were resulted in right hemiparesis, and ten in left hemiparesis. They had experienced the acute event at least one year prior to the study (mean time from onset of neurological damage 24 months). Inclusion criteria were: (i) unilateral paresis, (ii) ability to understand and follow simple instructions, (iii) minimum ability to perform active movements, even through trunk compensation, using the shoulder and/or the elbow joints. Exclusion criteria were: (i) bilateral impairment, (ii) severe sensory deficits in the paretic upper limb, (iii) cognitive impairment or behavioural dysfunction that would influence the ability to comprehend or perform the experiment, (iv) inability to provide informed consent and (v) other current severe medical problems. All subjects were right-handed. The level of the upper limb impairment for each post-stroke patient at admission is specified in Table 1 (sixth column).

A group of 5 healthy subjects, age range 48-65 (mean age $58.4 \pm 6.6$ ), two man and three women, all right-handed, was recruited for comparison purposes.

Table 1

Post-stroke subjects characteristics

\begin{tabular}{|c|c|c|c|c|c|c|c|c|}
\hline ID & Age & $\mathrm{DH}$ & Pathology & AS & $\mathrm{CM}$ & MSS-SE admission & MSS-SE discharge & MSS-SE follow-up \\
\hline S01 & 63 & $\mathrm{R}$ & Hemorrhagic stroke & $\mathrm{L}$ & 4 & 15.4 & 16.2 & 16.0 \\
\hline S02 & 50 & $\mathrm{R}$ & Brain injury & $\mathrm{L}$ & 3 & 10.2 & 11.8 & 11.8 \\
\hline S03 & 63 & $\mathrm{R}$ & Ischemic stroke & $\mathrm{L}$ & 3 & 13.8 & 16.2 & 18.2 \\
\hline S04 & 61 & $\mathrm{R}$ & Hemorrhagic stroke & $\mathrm{R}$ & 3 & 9.6 & 14.2 & 13.4 \\
\hline S05 & 45 & $\mathrm{R}$ & Hemorrhagic stroke & $\mathrm{R}$ & 3 & 10.4 & 12.0 & 12.0 \\
\hline S06 & 62 & $\mathrm{R}$ & Ischemic stroke & $\mathrm{L}$ & 3 & 12.8 & 16.2 & 14.4 \\
\hline S07 & 53 & $\mathrm{R}$ & Hemorrhagic stroke & $\mathrm{R}$ & 3 & 14.4 & 17.8 & 17.8 \\
\hline S08 & 64 & $\mathrm{R}$ & Hemorrhagic stroke & $\mathrm{R}$ & 3 & 10.6 & 12.2 & 11.4 \\
\hline S09 & 57 & $\mathrm{R}$ & Hemorrhagic stroke & $\mathrm{L}$ & 3 & 8.8 & 11.4 & 11.4 \\
\hline $\mathrm{S} 10$ & 57 & $\mathrm{R}$ & Ischemic stroke & $\mathrm{R}$ & 4 & 10.4 & 11.6 & 11.6 \\
\hline $\mathrm{S} 12$ & 62 & $\mathrm{R}$ & Ischemic stroke & $\mathrm{L}$ & 3 & 12.2 & 13.6 & 13.6 \\
\hline $\mathrm{S} 12$ & 36 & $\mathrm{R}$ & Brain injury & $\mathrm{L}$ & 3 & 14.6 & 15.0 & 18.8 \\
\hline S13 & 33 & $\mathrm{R}$ & Ischemic stroke & $\mathrm{L}$ & 5 & 35.2 & 37.4 & 37.4 \\
\hline S14 & 50 & $\mathrm{R}$ & Brain injury & $\mathrm{L}$ & 4 & 28.2 & 31.0 & 30.8 \\
\hline S15 & 41 & $\mathrm{R}$ & Ischemic stroke & $\mathrm{L}$ & 3 & 17.6 & 20.2 & 18.0 \\
\hline S16 & 34 & $\mathrm{R}$ & Ischemic stroke & $\mathrm{R}$ & 3 & 9.2 & 11.2 & 11.0 \\
\hline S17 & 52 & $\mathrm{R}$ & Ischemic stroke & $\mathrm{R}$ & 3 & 13.2 & 13.6 & 13.4 \\
\hline
\end{tabular}

(R): Right; (L): Left; (DH): Dominant Hand; (AS): Affected Side; (CM): Chedoke-McMaster Stroke Assessment; (MSS-SE): Shoulder-Elbow Motor Status Score. 
The experimental protocol, described in subsections 2.2-2.4, was approved by the local ethics committee and each subject signed a consent form.

\subsection{Experimental setup}

The InMotion2 robotic system (Interactive Motion Technologies, Inc., Boston, MA, USA) was used for this study. This system is derived from the MITMANUS, a robot designed for clinical and neurological applications [14]. The robotic system (see Fig. 1) allows subjects to execute reaching movements in the horizontal plane. During the movements the device can assist or resist the subject's movements. Table 2 shows the robotic arm's characteristics. A monitor in front of the subject displays the exercises to be performed. A second monitor is dedicated to the operator. The workstation is mounted on a custom-made adjustable chair, which allows the chair to be rotated $360^{\circ}$ and translated $0.5 \mathrm{~m}$ toward a table-top, specially designed to facilitate transfer of wheelchair-bound patients. A block

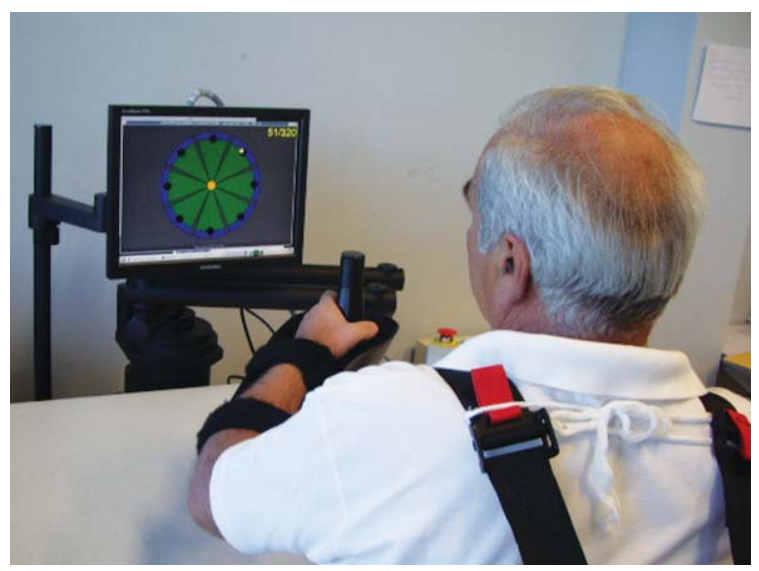

Fig. 1. A subject during the robot-aided therapy.

Table 2

Robotic arm's characteristic

\begin{tabular}{ll}
\hline DoFs & 2 \\
Inertia & $1 \mathrm{~kg} \pm 0.33$ \\
& Maximum anisotropy \\
& $2: 1$ \\
Friction & $0.84 \mathrm{~N} \pm 0.28$ \\
& Maximum anisotropy \\
& $2: 1$ \\
Forces & $0-45 \mathrm{~N}$ \\
Impedances & $0-2 \mathrm{~N} / \mathrm{mm}$ \\
\hline
\end{tabular}

(DoFs): Degrees of freedom. diagram describing the experimental setup is shown in Fig. 2.

The robot can move, guide, or perturb the movement of the upper limb of the subjects and can record endeffector kinematics and mechanical quantities such as the position, velocity, and applied forces. A 6 axis force/torque transducer is placed on the end-effector. The subject's arm was placed in a customized arm support attached to the end-effector of the robot arm.

\subsection{Intervention}

Each subject was asked to perform goal-directed, planar reaching tasks, which emphasized shoulder and elbow movements, moving from the centre target to each of 8 peripheral targets.

In each session subjects received 45 minutes of robot-mediated therapy, 3 sessions per week for 6 weeks. The robotic therapy was composed of 2 different kinds of exercises, unassisted (Record) and assisted movements (Adaptive), based on 8 targets placed around a circumference and a centre target (see Fig. 3).

In detail:

- Record: a series of 16 unassisted clockwise repetitions to each robot target. The goal is to reach toward each of the red targets shown on the monitor in front the patient and placed around a circumference. If the patient is able to reach the respective targets, the robot prompts him or her to move toward the next one. The patient is invited to complete one set around the circle in a clockwise fashion. In the event that the patient is unable to reach the target, the therapist pauses the device and moves the patient's arm passively to the next start position.

- Adaptive: a series of 320 assisted clockwise repetitions to each robot target. The robot prepositions the patient's arm at the centre target when the programme is activated. A visual performance display appears following 5 series of clockwise repetitions. This is an exercise programme that is adaptive in nature. Based on the patient's performance, the programme either increases or decreases the assistance provided to reach the targets, using an "impedance" control strategy.

Each session is formed by $(i)$ a series of 16 assisted clockwise repetitions to each robot target (training 


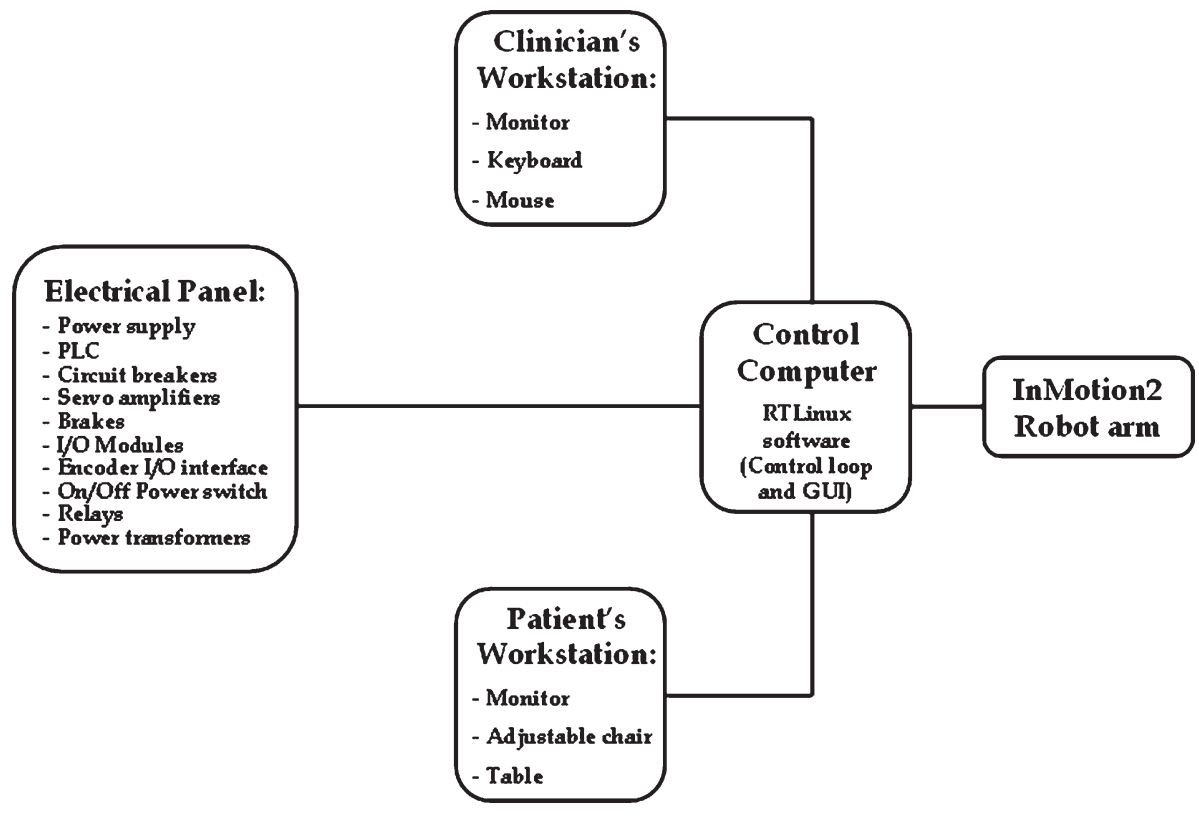

Fig. 2. Block diagram of the experimental setup.

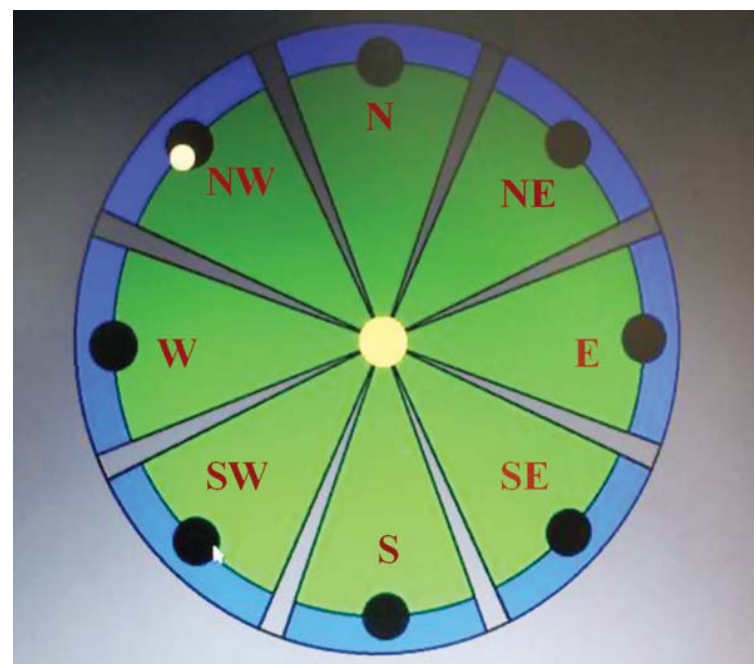

Fig. 3. The "clock-like" robotic therapy rehabilitative scenario.

test); (ii) a series of 16 unassisted clockwise repetitions to each robot target (Record); (iii) 3 series of assisted clockwise repetitions (Adaptive). At the end of each Adaptive series, the patient is asked to perform a series of 16 unassisted clockwise movements (Record).

The type of the movement used in the proposed robot-aided therapy induces the active mobilization of both the shoulder and the elbow joint, thus eliciting the use of the upper limb kinematic chain. The repetition of goal-directed planar movements can contribute to the motor recovery, in terms of improved control.

Biomechanical data were recorded from the robotic system before starting the robotic therapy and at the end of the therapy (after six weeks), during the Record series of exercises. Healthy subjects performed three series of the Record exercises in a single session.

\subsection{Outcome measures}

Each subject underwent an upper limb evaluation by an experienced physiatrist using the following scales:

- Stage of Arm section of the Chedoke-McMaster (CM) Stroke Assessment Scale: an evaluation tool that has high inter- and intra-rater repeatability, as well as strong correlation with the Fugl-Meyer (FM) score $[11,12]$.

- Motor Status Scale (MSS): which measures shoulder, elbow (maximum score $=40$ ), wrist, hand, and finger movements (maximum score $=42$ ). The MSS expands the measurement of upper extremity impairment and disability provided by the FM score and affords a reliable and valid assessment of upper limb impairment and disability following stroke [9]. The Motor Status Scale for shoulder and elbow (MSS-SE) was administered to the subjects. 
- Passive range of motion (ROM) in 11 different muscle groups ( 7 for the shoulder and 4 for the elbow).

- Modified Ashworth Scale (MAS) [3]: to assess muscle spasticity by rating resistance to passive stretch.

A common condition in neurologically impaired patients is pain in the shoulder joint [5]. The level of pain in the affected arm was assessed using a 4-point verbal rating scale $(0-3$, where 0 represents no pain, and 3 represents maximum pain) [7]

The level of feedback is critical to the success of patients. During the initial robot experiences (first 3 training sessions), it was important to review procedures and assess the level of understanding of each patient. A description of each performance measure was provided with the score.

Upon demonstration of competency and understanding by the patient, minimal feedback was provided. Verbal encouragement and environmental distraction was kept to a minimum.

A follow-up was carried out after 3 months. The same evaluation tools were used for each subject before (Pre-treatment), after (Post-treatment) the robotic therapy and in the follow-up phase. For statistical analysis a Wilcoxon signed-rank test was used.

\subsection{Parameters measuring movement dynamics}

All the gathered recordings represent a large amount of raw biomechanical data that should be processed in order to capture relevant characteristic features with respect to stroke patient recovery. Every recording contains discrete-time trajectories of position, velocity and forces with respect to three orthogonal directions. The mechanism is driven by brushless motors rated to $9.65 \mathrm{Nm}$ of continuous stall torque with 16-bit virtual absolute encoders for position and velocity measurements. Redundant velocity sensing may be provided by DC-tachometers with a sensitivity of $1.8 \mathrm{~V} / \mathrm{rad} / \mathrm{sec}$. The forces were measured by using the 6 axis force/torque transducer placed on the endeffector.

The robot's data were sampled at $200 \mathrm{~Hz}$. The measurements have been low-pass filtered at $40 \mathrm{~Hz}$ in order to reduce noise effect. The cut-off frequency was selected taking into account that human muscles can generate mechanical signals up to a maximum frequency of $40 \mathrm{~Hz}$ [21].
Let define $F_{\mathrm{x}}[k], F_{\mathrm{y}}[k]$ and $F_{\mathrm{Z}}[k]$ as the discrete-time force signals along the three orthogonal axes $x, y$ and $z$. It is assumed that effort direction is a relevant feature of the volitional movement [27].

Moreover, it has been demonstrated that in patients with chronic hemiparesis movements performed using the paretic limb are systematically misdirected [2].

The mean force vector is defined by its components, $\bar{F}_{x}, \bar{F}_{y}$ and $\bar{F}_{z}$, where

$$
\begin{aligned}
& \bar{F}_{x}=\frac{1}{N} \sum_{k=1}^{N} F_{x}[k] \\
& \bar{F}_{y}=\frac{1}{N} \sum_{k=1}^{N} F_{y}[k] \\
& \bar{F}_{z}=\frac{1}{N} \sum_{k=1}^{N} F_{z}[k]
\end{aligned}
$$

Given a recording, we compute the mean force direction features as the colatitude and azimuth angles of the mean force vector with respect to its referential (see Fig. 4), on a time window $t=1 \sec (N=200)$.

The colatitude $\phi_{F}$ is the angle between the $z$-axis and the mean force vector. The azimuth $\theta_{F}$ is the angle between the positive $x$-axis and the line from the origin to the end of the mean force vector projected onto the

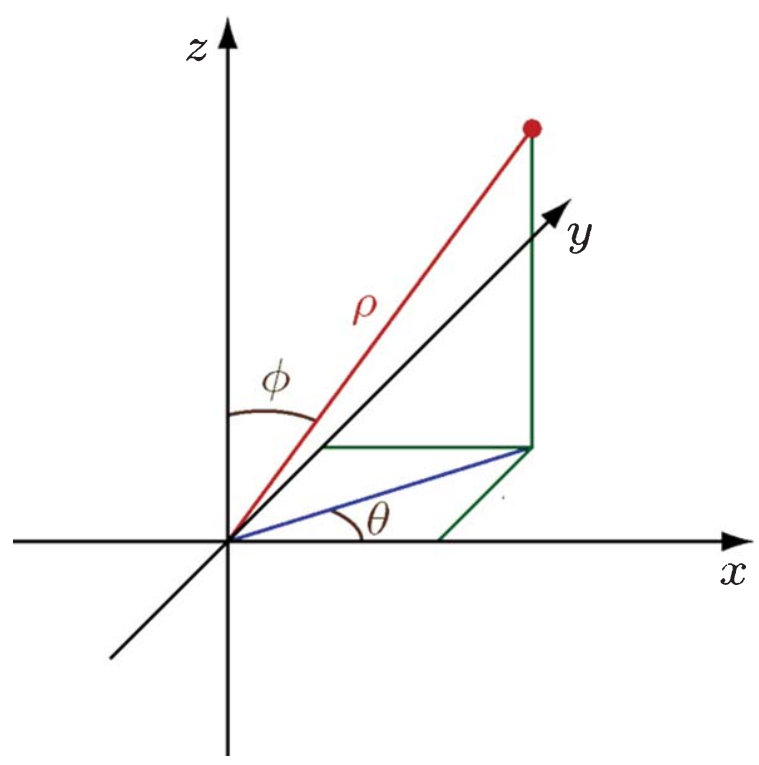

Fig. 4. The coordinate system showing the mean force vector $(\rho)$, the colatitude $(\phi)$ and the azimuth $(\theta)$ angles. 
$x y$-plane. These angles are obtained by converting the cartesian coordinates of the mean force to spherical coordinates.

$$
\begin{aligned}
& \rho=\sqrt{\bar{F}_{x}^{2}+\bar{F}_{y}^{2}+\bar{F}_{z}^{2}} \\
& \phi_{F}=\arccos \left(\frac{\bar{F}_{z}}{\rho}\right) \\
& \theta_{F}=\arctan \left(\frac{\bar{F}_{y}}{\bar{F}_{x}}\right)+\pi u_{0}\left(\bar{F}_{x}\right) \operatorname{sgn}\left(\bar{F}_{y}\right)
\end{aligned}
$$

where $u_{0}()$ stands for the Heaviside unit step function

$$
u_{0}(x)= \begin{cases}0 & \text { if } x \leq 0 \\ 1 & \text { if } x>0\end{cases}
$$

and $\operatorname{sgn}()$ denotes the signum function

$$
\operatorname{sgn}(x)=\left\{\begin{array}{cc}
-1 & \text { if } x<0 \\
0 & \text { if } x=0 \\
1 & \text { if } x>0
\end{array}\right.
$$

The angular deviation of every effort sample within the analysis frame from the mean effort is computed. The underlying hypothesis relies on the consideration that trajectories in pathological subjects could show larger deviations from the mean direction than in normal controls: the distribution of these angular deviations depicts some specific pattern (sudden variations, lack of smoothness, etc.) in the stroke patients movements.

Based on the rehabilitation scenario described in Fig. 2, each subject is asked to perform 16 different directions of movement: $\mathrm{N}_{\mathrm{t}}, \mathrm{N}_{\mathrm{b}}, \mathrm{NE}_{\mathrm{t}}, \mathrm{NE}_{\mathrm{b}}, \mathrm{E}_{\mathrm{t}}, \mathrm{E}_{\mathrm{b}}, \mathrm{SE}_{\mathrm{t}}$, $\mathrm{SE}_{\mathrm{b}}, \mathrm{S}_{\mathrm{t}}, \mathrm{S}_{\mathrm{b}}, \mathrm{SW}_{\mathrm{t}}, \mathrm{SW}_{\mathrm{b}}, \mathrm{W}_{\mathrm{t}}, \mathrm{W}_{\mathrm{b}}, \mathrm{NW}_{\mathrm{t}}, \mathrm{NW}_{\mathrm{b}}$. The subscript $t$ stands for "toward" (i.e., the movement from the center to the specific peripheral target), $b$ stands for "back" (i.e., the movement from the specific peripheral target to the center). Given a recording, the angular deviation $\delta_{F}[k]$ between the $k$-th force sample $\left(F_{x}[k]\right.$, $\left.F_{y}[k], F_{z}[k]\right)$, within the analysis frame $k=1, \ldots, N$, and the mean force $\left(\bar{F}_{x}, \bar{F}_{y}, \bar{F}_{z}\right)$ is computed as the inverse cosine of the normalized scalar product.

$$
\begin{aligned}
& \vec{a}=\left(\bar{F}_{s, x}, \bar{F}_{s, y}, \bar{F}_{s, z}\right) \\
& \vec{b}=\left(F_{x}[k], F_{y}[k], F_{z}[k]\right) \\
& \delta_{F}[k]=\arccos \left(\frac{\vec{a}}{\|\vec{a}\|} \cdot \frac{\vec{b}}{\|\vec{b}\|}\right)=\arccos \left(\frac{\bar{F}_{x} F_{x}[k]+\bar{F}_{y} F_{y}[k]+\bar{F}_{z} F_{z}[k]}{\sqrt{\bar{F}_{x}^{2}+\bar{F}_{y}^{2}+\bar{F}_{z}^{2}} \sqrt{F_{x}[k]^{2}+F_{y}[k]^{2}+F_{z}[k]^{2}}}\right)
\end{aligned}
$$

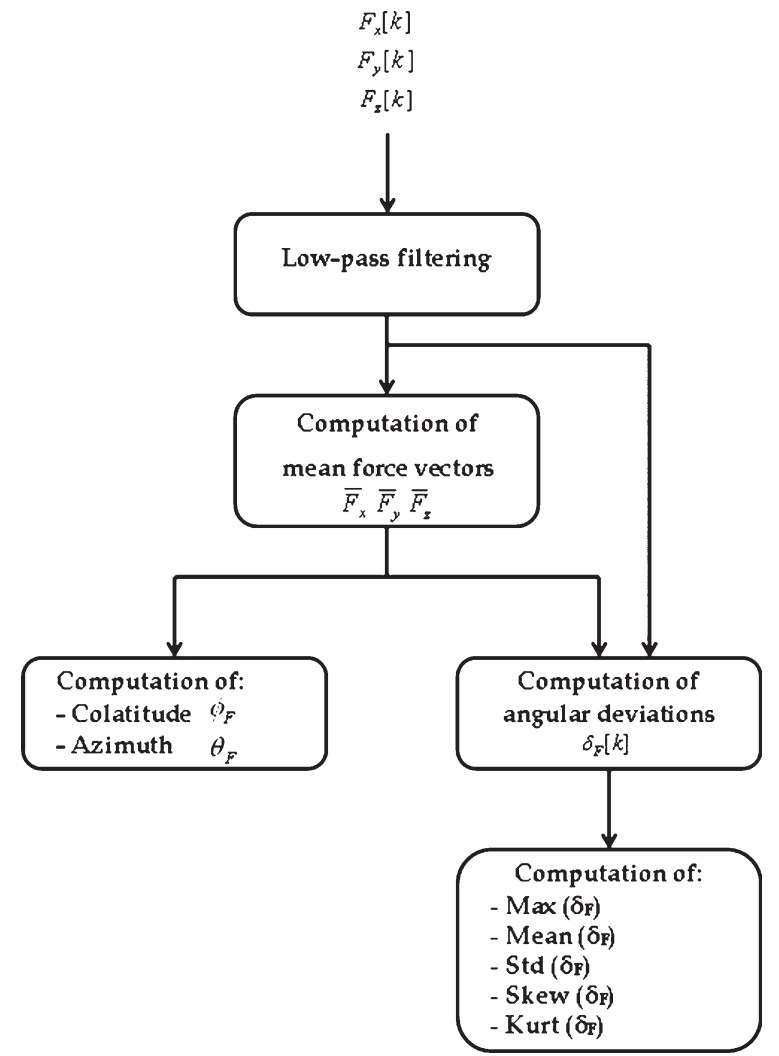

Fig. 5. Flowchart of the proposed method of force metric.

Five different features are computed in order to characterize the distribution of the angular deviations $\delta_{F}[k], k=1, \ldots, N$. The angular deviations can take values between 0 to $\pi$. The maximum value $\operatorname{Max}\left(\delta_{F}\right)$ is computed in order to characterize the support of the distribution. The mean value $\operatorname{Mean}\left(\delta_{F}\right)$ and the standard deviation $\operatorname{Std}\left(\delta_{F}\right)$ are estimated in order to characterize the central tendency and the dispersion of the distribution, respectively. Moreover, the skewness $\operatorname{Skew}\left(\delta_{F}\right)$ and the kurtosis $\operatorname{Kurt}\left(\delta_{F}\right)$ are estimated in order to characterize the asymmetry and the peakedness of the distribution. Figure 5 shows a flowchart describing the proposed method. Data were processed using custom routines developed under the Matlab environment 


$$
\begin{aligned}
\operatorname{Max}\left(\delta_{F}\right)= & \underset{k=1, \ldots, N}{\arg \max }\left(\delta_{F}[k]\right) \\
\operatorname{Mean}\left(\delta_{F}\right)= & \frac{1}{N} \sum_{k=1}^{N} \delta_{F}[k] \\
\operatorname{Std}\left(\delta_{F}\right)= & \left.\frac{1}{N-1} \sum_{k=1}^{N}\left(\delta_{F}[k]-\operatorname{Mean}\left(\delta_{F}\right)\right)^{2}\right)^{1 / 2} \\
\operatorname{Skew}\left(\delta_{F}\right)= & \frac{n}{(n-1)(n-2)} \\
& \times \frac{\sum_{k=1}^{N}\left(\delta_{F}[k]-\operatorname{Mean}\left(\delta_{F}\right)\right)^{3}}{\left(\sum_{k=1}^{N}\left(\delta_{F}[k]-\operatorname{Mean}\left(\delta_{F}\right)\right)^{2}\right)^{3 / 2}}
\end{aligned}
$$

The mean acceleration $\bar{a}$ was computed, following a similar procedure.

The Trajectory Error (TE) is defined as a normalized difference between the desired trajectory and the patient's trajectory from one point in the workspace to another. Desired trajectory is considered as a straight line from the starting target to the final target. Absolute values of the deviations from this straight line trajectory during the point-to-point movement were summed to obtain the non-normalized value of this parameter. To normalize it with respect to time, such value was divided by the total number of samples recorded during the specific movement. Then it was divided by the distance from the starting point to the final point of the movement in order to obtain spatial normalization.

$\operatorname{Kurt}\left(\delta_{F}\right)=\frac{n(n+1)}{(n-1)(n-2)(n-3)} \frac{\sum_{k=1}^{N}\left(\delta_{F}[k]-\operatorname{Mean}\left(\delta_{F}\right)\right)^{4}}{\left(\sum_{k=1}^{N}\left(\delta_{F}[k]-\operatorname{Mean}\left(\delta_{F}\right)\right)^{2}\right)^{2}}-3 \frac{(n-1)^{2}}{(n-2)(n-3)}$

These parameters can be interpreted as measures providing a quantitative description of the distribution of the mean force vector.

The following biomechanical parameters were computed: 1) mean velocity, 2) mean acceleration and 3) mean trajectory error.

For each subject, the velocities $v_{x}[k]$ and $v_{y}[k]$, defined as the discrete-time velocity signals along $x$ and $y$ axes, respectively, were recorded for each movement in the Record series.

In this study, the resultant velocity in the $x-y$ plane was considered: for each movement such variable is defined by its components $v_{x}[k]$ and orthogonal $v_{y}[k]$, as follows:

$$
v_{x y}[k]=\sqrt{v_{x}[k]^{2}+v_{y}[k]^{2}}
$$

For each movement, the mean speed is defined as follows:

$$
\overline{v_{x y}}=\frac{1}{N} \sum_{k=1}^{N} v_{x y}[k]
$$

where $N$ represents the number of samples for each recording. On each Record series, an average of the values of the mean speed among the 16 directions was computed.

A mean value $\bar{v}$ among the different averages of the Record series was computed as well: it represents the overall mean speed for the specific session of the robotaided therapy.
This final value, normalized both spatially and temporally, is a dimensionless value and represents the final TE value for the movement.

For each session, the mean Trajectory Error $(\overline{T E})$ is computed as the mean value among the different averages of the Record series on the 16 directions.

Moreover, the percentage of help provided by the robot along the motion during the Adaptive series was computed using two parameters, named Initiated movement and Distance along straight line.

The former indicates the percentage of time the patient is able to initiate a movement without robot assistance. A score of $100 \%$ infers the patient initiated each movement independently. This parameter highlights whether the patient made any movement, in any direction, during the first 2 seconds of each requested movement.

The latter measures the relative accuracy of movement to reach the desired targets. A score of $100 \%$ infers the patient completed each movement accurately without robot assistance to minimize deviation of trajectory. This parameter points out whether the patient could reach the target or not.

\section{Results}

The robot-aided therapy was well accepted and tolerated by the patients. Moreover, this type of training 
contributed to increase the motivation of the patients during the rehabilitation period.

The results from outcome measures show a significant decrease in motor impairment in the paretic upper limb after the treatment. As shown in Table 3, statistically significant improvements were found on the MSS-SE measured before and after the robotic treatment $(W=153.00, \mathrm{~T}+=153.00, \mathrm{~T}-=0.00 ; p<0.001)$.

The MSS-SE at the follow-up evaluation confirmed that the motor improvements were kept after 3 months. No statistically significant changes were observed between MMS-SE at the end of the treatment and at 3 months follow-up $(W=-19.00, \mathrm{~T}+=18.00$, $\mathrm{T}-=-37.00$; not significant $p>0.05)$.

In the MAS, the sum of muscles trained in the shoulder (7 muscles) was considered. The shoulder MAS score decreased significantly after the training $(W=-112.00, \mathrm{~T}+=4.00, \mathrm{~T}-=-116.00 ; p<0.001)$. No modifications in the shoulder MAS score were found at follow-up $(W=-34.00, \mathrm{~T}+=16.00, \mathrm{~T}-=$ -50.00 ; not significant $p>0.05)$. The change in the elbow MAS score after the training was not statistically significant $(W=-43.00, \mathrm{~T}+=17.50, \mathrm{~T}-=-60.50$; not significant $p>0.05)$.

The seven parameters described in the previous section $\left(\operatorname{Max}\left(\delta_{F}\right), \operatorname{Mean}\left(\delta_{F}\right), \operatorname{Std}\left(\delta_{F}\right), \operatorname{Skew}\left(\delta_{F}\right), \operatorname{Kurt}\left(\delta_{F}\right)\right.$, $\left.\phi_{F}, \theta_{F}\right)$ were computed for each subject $\left(i_{h}=1, \ldots, 5\right.$ for the healthy subjects, $i_{p s}=1, \ldots, 17$ for the post-stroke subjects) and for two directions: $\mathrm{N}_{\mathrm{t}}$ and $\mathrm{S}_{\mathrm{b}}$. The two directions represent the planar movement (from south target to the centre, from the centre to the north target) which offers the maximum resistance to the pathological pattern of such subjects, characterized by a flexion of the elbow joint.

For the post-stroke subjects, the parameters were computed before starting the robot-aided upper limb rehabilitation (Pre-treatment) and after its completion
(Post-treatment). Therefore, three different matrices $\mathrm{P}_{\mathrm{H}}, \mathrm{P}_{\mathrm{PSPRE}}, \mathrm{P}_{\mathrm{PSPOST}}$ (where H=healthy, PSPRE=PostStroke Pre-treatment, PSPOST=Post-Stroke Posttreatment) containing the proposed parameters were computed.

Among the different combination of pairs direction/parameter, the t-test performed on the difference between $P_{\text {PSPRE }}$ and $P_{\text {PSPOST }}$ resulted in a statistical significance only for Skew $\left(\delta_{F}\right) / \mathrm{N}_{\mathrm{t}}(p<0.005)$ and $\operatorname{Skew}\left(\delta_{F}\right) / \mathrm{S}_{\mathrm{b}}(p<0.05)$.

Based on these results, our hypothesis has been demonstrated: the direction $\mathrm{S}_{\mathrm{b}}-\mathrm{N}_{\mathrm{t}}$ represents the most difficult planar reaching movement to be performed by a spastic stroke subject, as it opposes to the elbow flexion pathological pattern, characteristic of this type of patient. Moreover, the skewness of the distribution of the deviations from the mean force vector can discriminate the patients between the starting and the end of the robot-aided treatment.

Deviations from the mean force vector in healthy subjects and in post-stroke patients before and after the robotic therapy, in the direction $\mathrm{S}_{\mathrm{b}}, \mathrm{N}_{\mathrm{t}}, \mathrm{W}_{\mathrm{b}}$ and $\mathrm{E}_{\mathrm{t}}$ are shown in Fig. 6a, b, c and d, respectively.

Figures 7 and 8 show the $\operatorname{Mean}\left(\delta_{F}\right)$ for direction $\mathrm{S}_{\mathrm{b}}$ and the $\operatorname{Max}\left(\delta_{F}\right)$ for direction $\mathrm{N}_{\mathrm{t}}$, respectively, in $\mathrm{P}_{\mathrm{H}}$, PPSPRE and PPSPOST.

For both variables, post-stroke patients at the end of the treatment show a value approaching that from healthy subjects.

Figures 9 and 10 show the $\operatorname{Mean}\left(\delta_{F}\right)$ for direction $\mathrm{S}_{\mathrm{b}}$ and the $\operatorname{Max}\left(\delta_{F}\right)$ for direction $\mathrm{N}_{\mathrm{t}}$, respectively, in each post-stroke subject, before and at the end of the robot-aided treatment, together with the mean value of these parameters computed on the data from healthy subjects.

The mean speed, mean acceleration and mean trajectory error are shown in Figs 11, 12 and 13, respectively.

Table 3

Outcome measures comparison at admission and discharge

\begin{tabular}{|c|c|c|c|c|c|}
\hline \multirow[t]{2}{*}{ Evaluation } & \multicolumn{2}{|c|}{ Admission } & \multicolumn{2}{|c|}{ Discharge } & \multirow[t]{2}{*}{$p$} \\
\hline & Median & IQR & Median & IQR & \\
\hline MSS-SE & 12.800 & $10.350-14.800$ & 14.200 & $11.950-16.600$ & $<0.001$ \\
\hline MAS shoulder & 8.000 & $4.750-11.250$ & 4.000 & $2.750-6.625$ & $<0.001$ \\
\hline MAS elbow & 1.500 & $0.750-2.000$ & 1.000 & $0-1.500$ & ns \\
\hline ROM shoulder [degrees] & 440.000 & $408.750-566.250$ & 550.000 & $477.500-647.500$ & $<0.001$ \\
\hline ROM elbow [degrees] & 440.000 & $417.500-460.000$ & 460.000 & $450.000-460.000$ & $<0.005$ \\
\hline
\end{tabular}

IQR: Interquartile Range; MAS: Modified Ashworth Scale; MSS-SE: Motor Status Score - Shoulder-Elbow; ns: not significant; ROM: range of motion. 

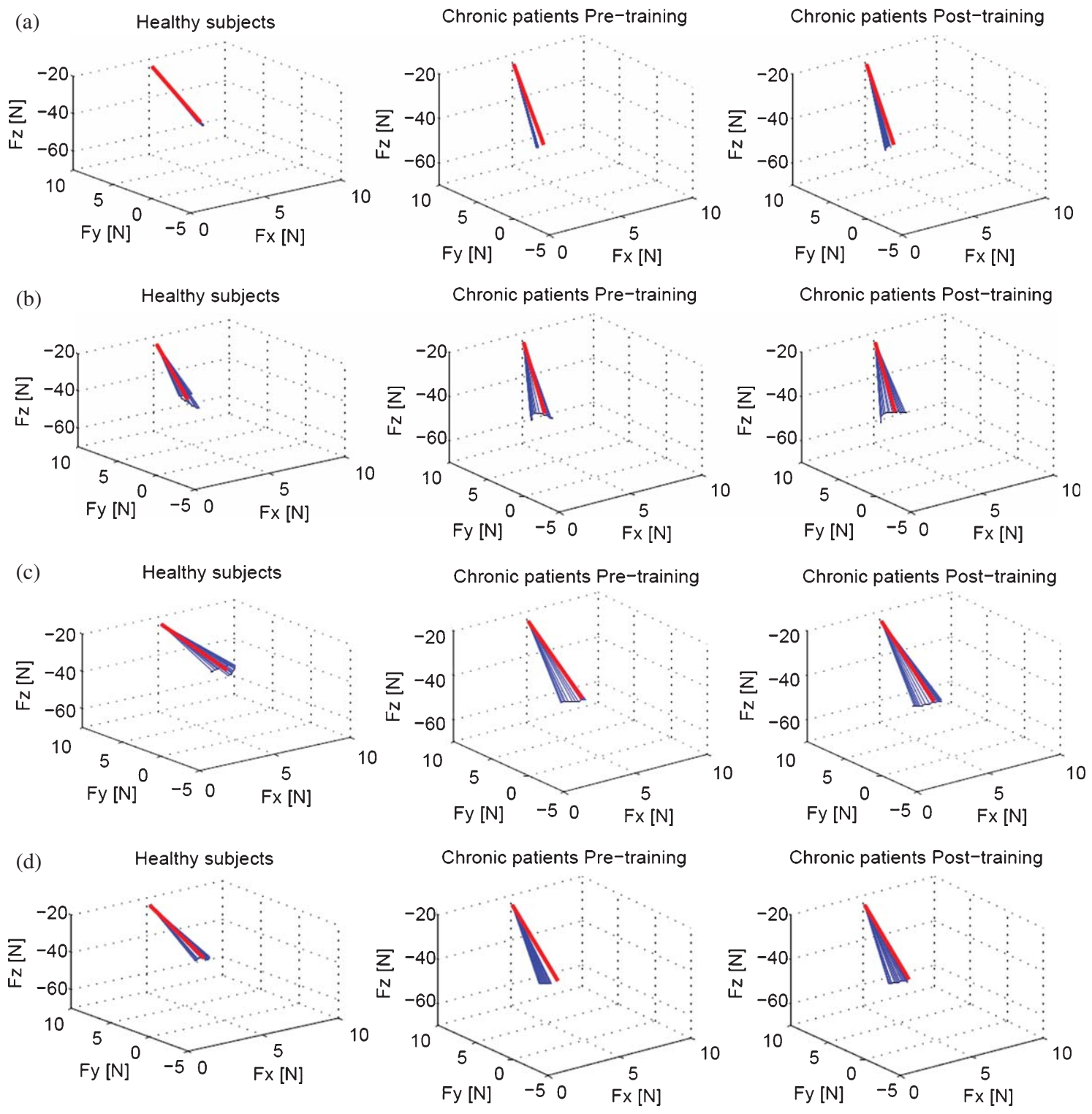

Fig. 6. Deviations from the mean force vector in healthy subjects and in a post-stroke patients: direction $N_{t}(a)$, direction $S_{b}(b)$, direction $W_{b}$ (c), direction $\mathrm{E}_{\mathrm{t}}(\mathrm{d})$.

Figures 14 and 15 show mean values of Initiated movement and Distance along straight line respectively, before and after the robot-aided rehabilitation.

\section{Discussion}

The paper presented a method for quantitative assessment of reaching movements in post-stroke patients. The method is based on the analysis of biomechanical parameters computed from force data recorded during the robot-aided rehabilitation, which can be used to quantify the motor recovery process of each patient.

Previous studies proposed a force metric for assessing the effects of a robot-aided in post-stroke subjects $[6,18,20]$.

The force directional error, measuring the directional error of the patient-exerted force applied to the end-effector of the robot device, is computed as mean 


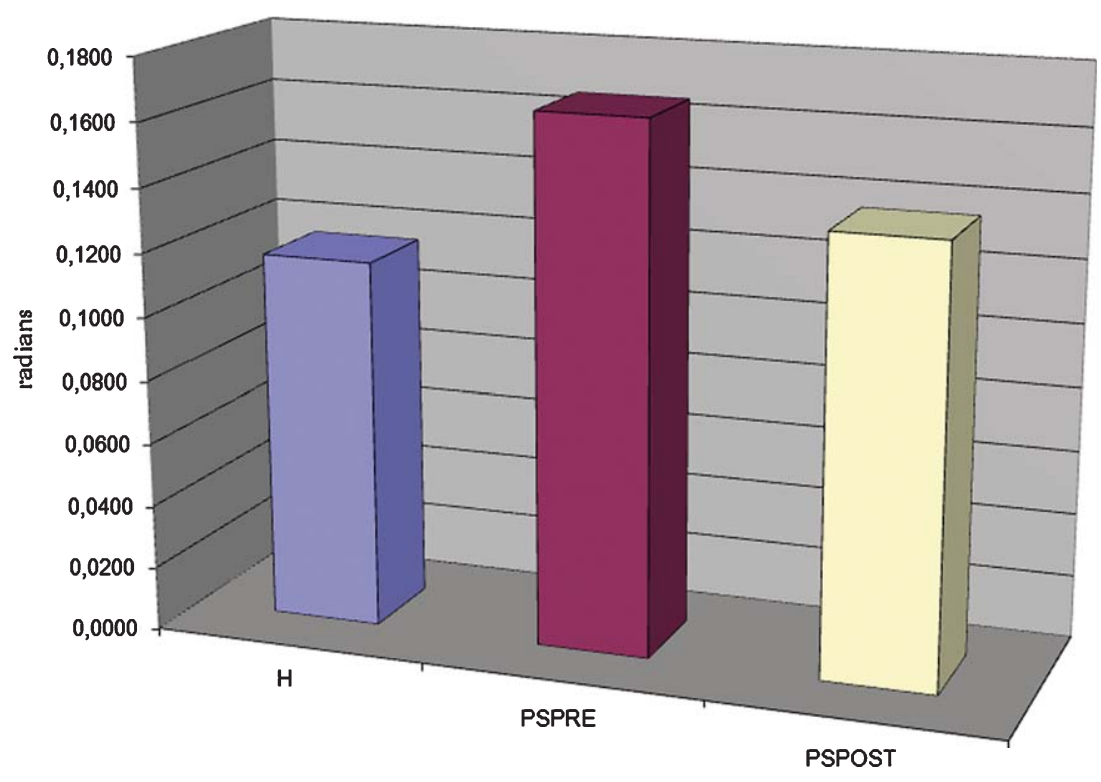

Fig. 7. Group values of parameter Mean $\left(\delta_{F}\right)$ for direction $S_{b}$, in $P_{H}, P_{P S P R E}$ and $P_{P S P O S T}$.

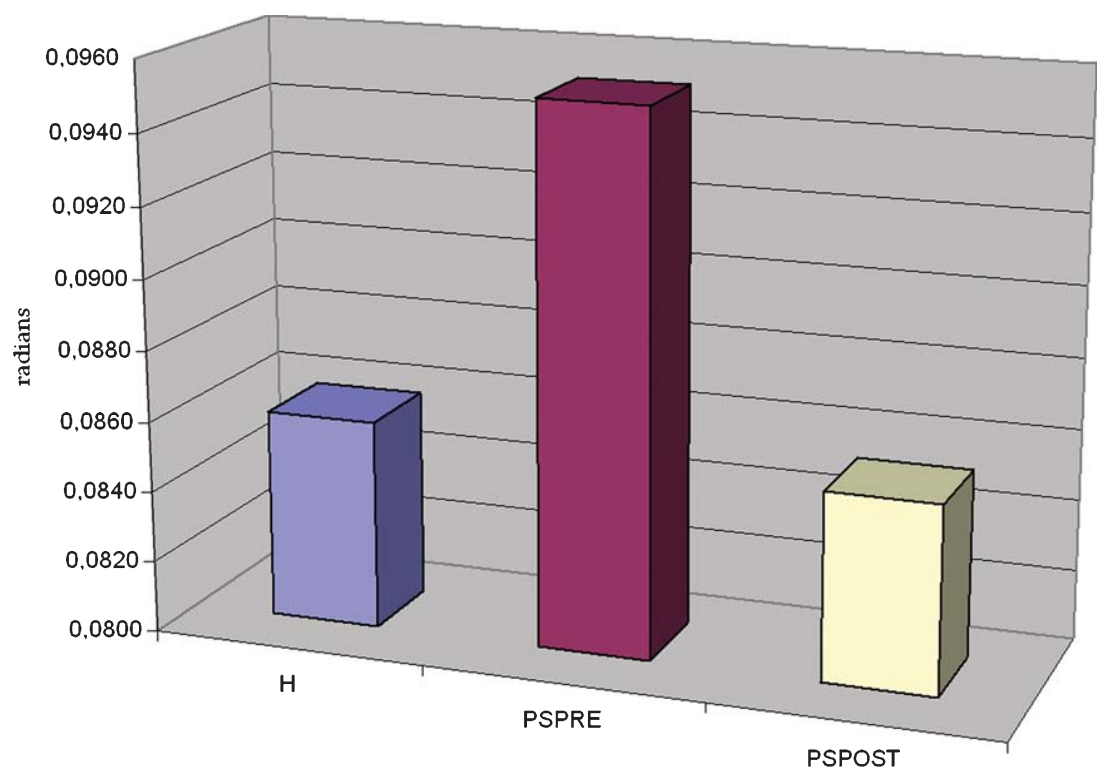

Fig. 8. Group values of parameter $\operatorname{Max}\left(\delta_{F}\right)$ for direction $\mathrm{N}_{\mathrm{t}}$, in $\mathrm{P}_{\mathrm{H}}, \mathrm{P}_{\mathrm{PSPRE}}$ and PPSPOST.

value from all reaching movements of the training session.

On the contrary, our method is based on the computation of the deviations from the mean force vector in each direction of the different reaching movements requested to the subjects.
Furthermore, the metric used in the above mentioned studies leaves undetected the information of which direction could be appropriate or not for a given reaching movement.

Our method allow to perform a detailed analysis of the reaching directions: it may contribute to the identi- 


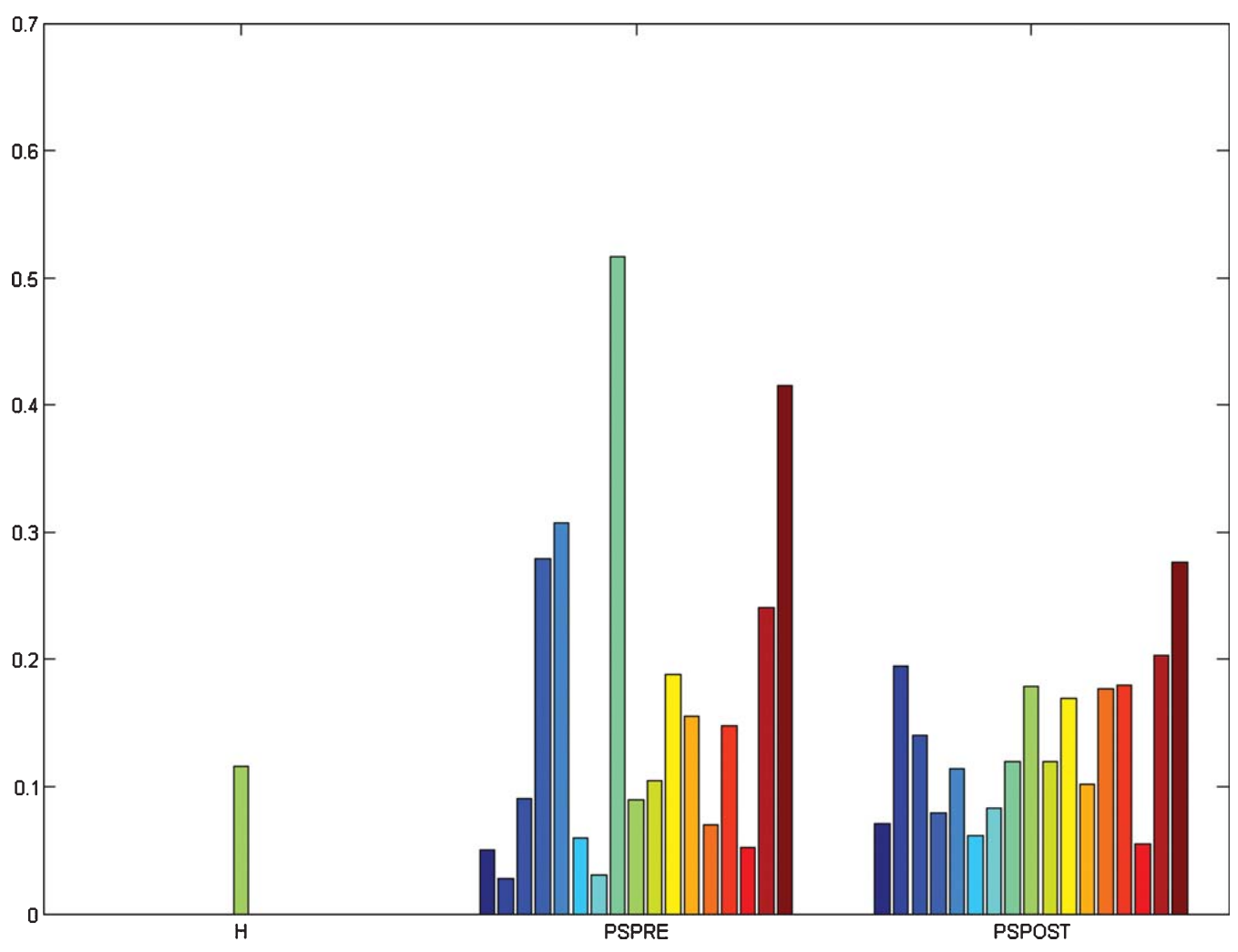

Fig. 9. Subjects's values of parameter Mean $\left(\delta_{F}\right)$ for direction $\mathrm{S}_{\mathrm{b}}$, in $\mathrm{P}_{\mathrm{H}}$, $\mathrm{P}_{\mathrm{PSPRE}}$ and PPSPOST (Y-axis units: radians).

fication of whether each subject show some privileged directions, if they are predictors of the upper limb motor recovery and at which extent they can promote it (Fig. 6).

The motor improvement in the upper limb impairment of post-stroke patients after the rehabilitation based on a robot-aided therapy has been demonstrated by several previous studies $[8,10,15,16,19,20,22$, $24,30]$.

The results here presented confirm such improvement (Table 3), which may be associated either to the appearance of compensation mechanisms or to a recovery of physiological upper limb movements. The former is mainly characterized by changes in the direction of force vectors, the latter by a process of re-learning of planar reaching movements, without significant changes compared to healthy subjects.

The improvement in the upper limb motor performance of chronic stroke patients recruited in this study is also confirmed by the values of (i) the mean speed, mean acceleration, mean Trajectory Error (Figs 11-13) and (ii) the two parameters associated to the reduced percentage of help provided by the robot along the motion during the Adaptive series, at the end of the therapy (Figs 14 and 15). Due to the nature of the adaptive control strategy implemented in the robotic system, such decrease is associated with an improved active upper limb movement.

The analysis of the different directions has revealed that in the group of post-stroke subjects the difference between the start and the end of the robot-aided therapy is statistically significant in direction $S_{b}$ and $\mathrm{N}_{\mathrm{t}}$.

Results presented in this paper, focused on these directions, show that the motor recovery of planar reaching movements is based on relearning mechanisms: in fact, the values of the maximum, the mean and the colatitude at the end of the treatment are similar to those from healthy subjects (Tables 4 and 5, columns $1,2,6)$.

Therefore, the motor recovery process of post-stroke subjects may be explained by means of the preservation of biomechanical and physiological properties in the upper limb. 


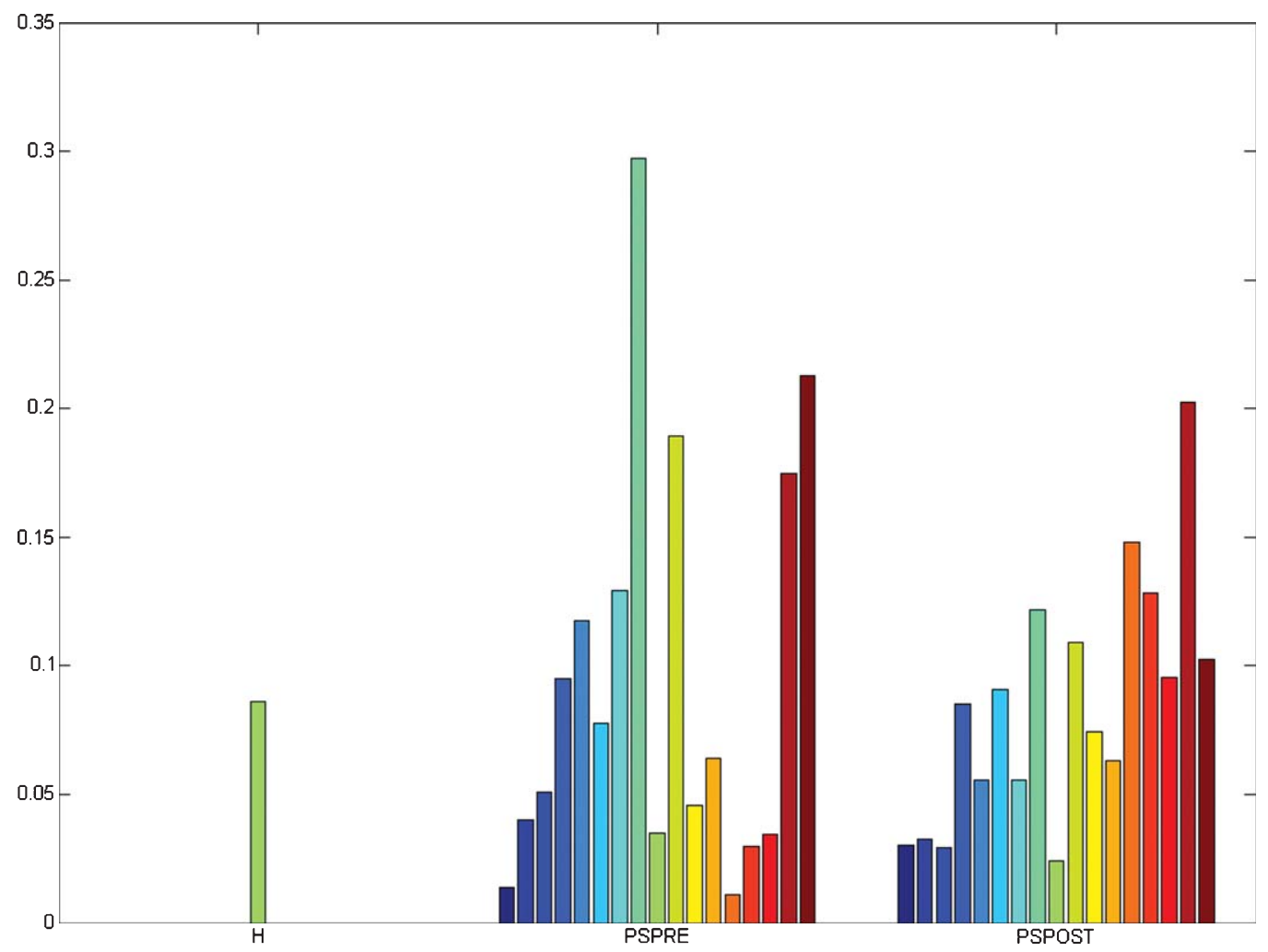

Fig. 10. Subjects's values of parameter $\operatorname{Max}\left(\delta_{F}\right)$ for direction $\mathrm{N}_{\mathrm{t}}$, in $\mathrm{P}_{\mathrm{H}}, \mathrm{P}_{\mathrm{PSPRE}}$ and PPSPOST (Y-axis units: radians).

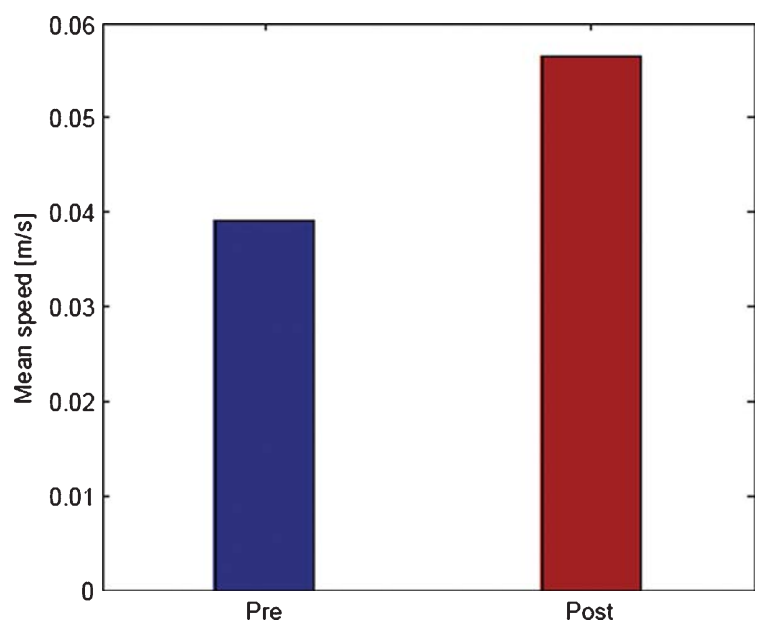

Fig. 11. Mean speed.

Moreover, the values of the parameters associated to the distribution of the deviations from the mean force vector (i.e. $\operatorname{Skew}\left(\delta_{F}\right)$ and $\operatorname{Kurt}\left(\delta_{F}\right)$ ), for direction $\mathrm{S}_{\mathrm{b}}$, at the end of the robot-aided therapy are close to those from the healthy subjects (Table 4, columns 4-5).

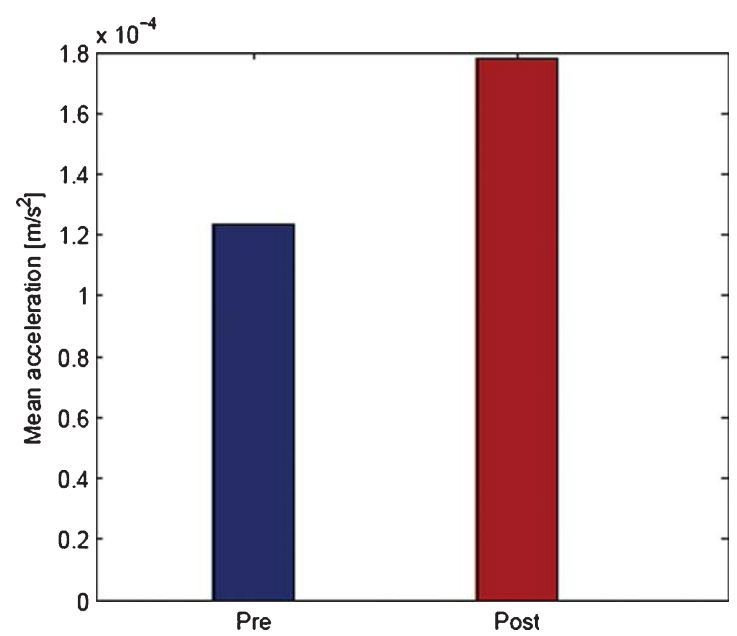

Fig. 12. Mean acceleration

The statistical significant change of $\operatorname{Skew}\left(\delta_{F}\right)$ in both directions in post-stroke patients could be linked to a more homogeneous distribution of the deviations from the mean force vector observed at the end of the treatment. 


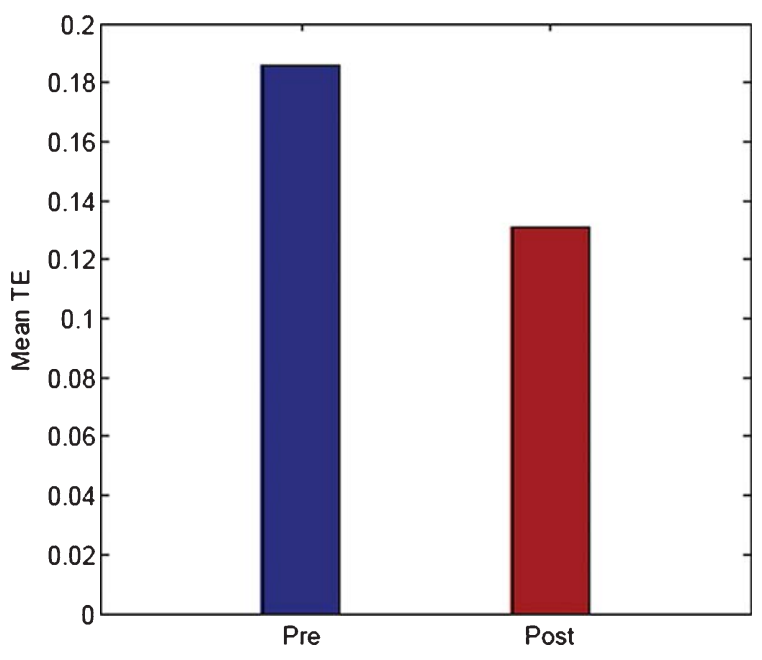

Fig. 13. Mean trajectory error.

In terms of the subject's ability to move in a desired direction, it can be interpreted as an improvement in the execution of the reaching movement from the $S_{b}$ to the $\mathrm{N}_{\mathrm{t}}$, due to the reduced dispersions of the deviations around the mean force vector, if compared to the start of the treatment. The other measures might not have reached significance due to the high inter-subject variability.

These results seem to show that during the time course of the robot-aided rehabilitation, the distribution of the deviations from the mean vector in the two privileged directions $\left(\mathrm{S}_{\mathrm{b}}\right.$ and $\mathrm{N}_{\mathrm{t}}$ ) moves towards that observed in the group of healthy subjects.
Moreover, in the group of post-stroke subjects (i) the mean value of the force deviations in direction $S_{b}$ and (ii) the maximum value in direction $\mathrm{N}_{\mathrm{t}}$ during the time course of the robotic treatment increase and move towards that from the healthy subjects group (Figs 7 and 8).

A possible explanation of this trend in post-stroke subjects can be described in terms of increasing requested force amplitude in direction $S_{b}$, due to the initial flexion of the elbow joint, and a progressive adjustment of the required force in direction $\mathrm{N}_{\mathrm{t}}$, as the movement proceeds towards the final target. This result can be explained taking into account that in our study, during the Adaptive series, a virtual slot, supporting the movement in the desired direction of each reaching movement and limiting as much as possible movements in undesired directions, was used.

The values of the computed parameters based on directional deviations from the mean force vector may provide both therapists and subjects with useful feedback. Moreover, for each patient, the comparison of such values during the time course of the robot-aided rehabilitation treatment with those from the healthy subjects gives important information about the motor performances and can detect motor recovery.

The analysis of the directional deviations during the training period conveys information which could be used to plan and, when necessary, drive the decision whether to continue, modify or stop the rehabilitation strategies The inclusion of our parameters providing information about the force control recovery of poststroke subjects into the rehabilitation procedures may

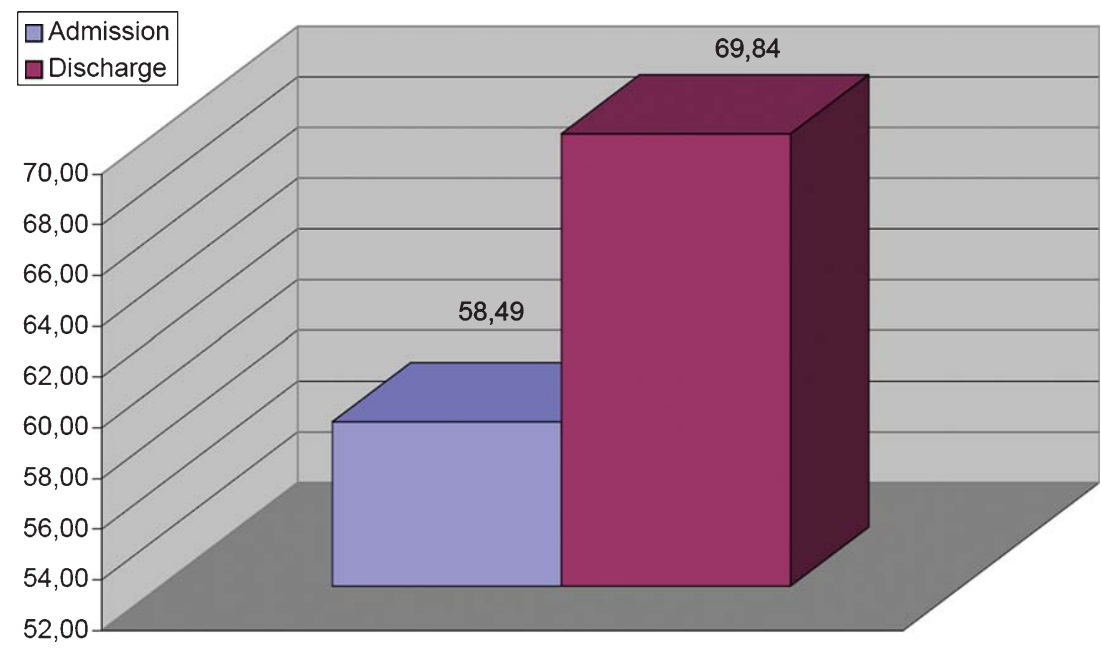

Fig. 14. Initiated movement. 


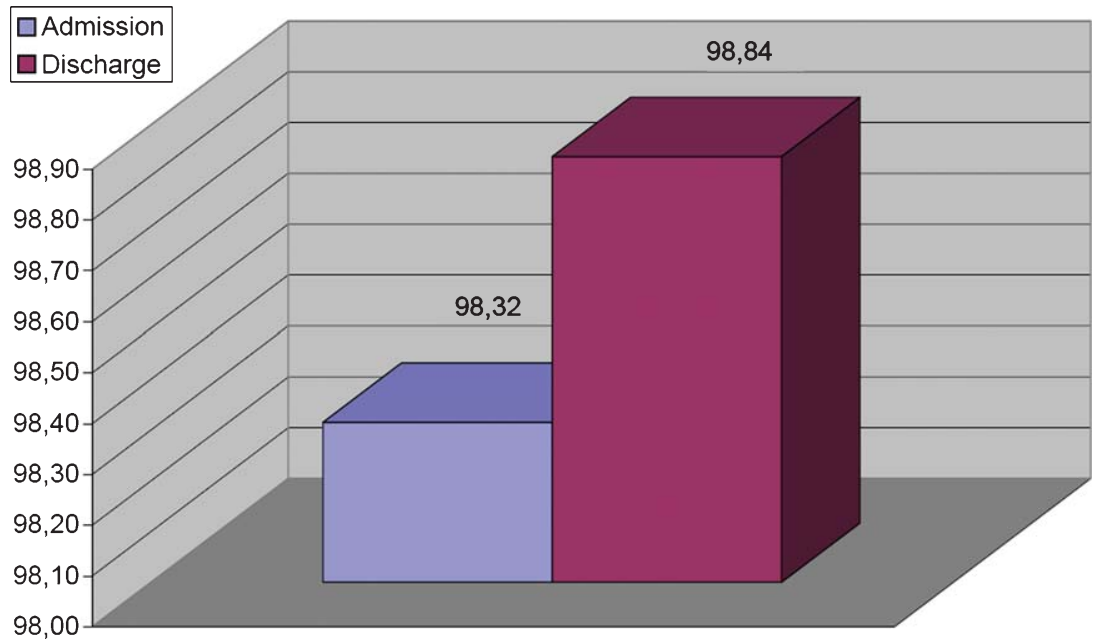

Fig. 15. Distance along straight line.

Table 4

Parameters in Healthy (H), Post-stroke subjects before (PS $\mathrm{PRE}_{\text {) and after (PS }}$ POST) the robot-aided rehabilitation, for direction $\mathrm{S}_{\mathrm{b}}$ (units column 1-3 and column 6-7: radians; column 4-5: adimensional)

\begin{tabular}{lccccccc}
\hline & $\operatorname{Max}\left(\delta_{F}\right)$ & Mean $\left(\delta_{F}\right)$ & $\operatorname{Std}\left(\delta_{F}\right)$ & Skew $\left(\delta_{F}\right)$ & Kurt $\left(\delta_{F}\right)$ & $\phi_{F}$ & $\theta_{F}$ \\
\hline $\mathrm{H}$ & 0.3624 & 0.1162 & 0.1006 & 1.0860 & 3.1532 & 3.0217 & 0.7095 \\
PS & 0.3401 & 0.1661 & 0.0919 & 0.4100 & 2.5081 & 3.0296 & 0.8347 \\
PS & 0.3439 & 0.1366 & 0.0874 & 0.7072 & 3.1646 & 3.0248 & 0.8706 \\
\hline
\end{tabular}

Table 5

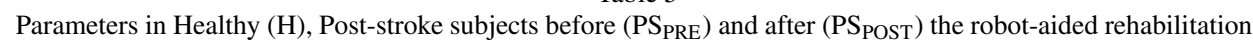
for direction $\mathrm{N}_{\mathrm{t}}$ (units column 1-3 and column 6-7: radians; column 4-5: adimensional)

\begin{tabular}{lccccccc}
\hline & $\operatorname{Max}\left(\delta_{F}\right)$ & $\operatorname{Mean}\left(\delta_{F}\right)$ & $\operatorname{Std}\left(\delta_{F}\right)$ & Skew $\left(\delta_{F}\right)$ & $\operatorname{Kurt}\left(\delta_{F}\right)$ & $\phi_{F}$ & $\theta_{F}$ \\
\hline $\mathrm{H}$ & 0.0859 & 0.0503 & 0.0214 & -0.5793 & 2.6092 & 3.0234 & 0.4617 \\
PS & 0.0952 & 0.0325 & 0.0230 & 0.5162 & 3.0913 & 3.0432 & 0.6903 \\
PS & 0.0853 & 0.0344 & 0.0194 & 0.6052 & 3.3700 & 3.0390 & 0.7639 \\
\hline
\end{tabular}

contribute to perform a proper tuning of the training, evaluate the effects of the robotic therapy and tailor the robot-aided rehabilitation treatment to the actual residual abilities of each subject.

The upper limb motor improvement shown by our preliminary results should be confirmed by an improved muscle activation patterns at the end of the robotic therapy. Ongoing studies based on the analysis of electromyographic recordings from the upper limb muscles of post-stroke subjects during the robot-aided treatment will contribute to highlight a more detailed model of the motor recovery.

We also observed that the robot-aided therapy, based on an adaptive control strategy, shows different effects on the post-stroke subjects, based on their upper limb severity (Figs 9 and 10): in fact, during the Adaptive series of exercises, based on each subject's perfor- mance, the assistance provided by the robotic system to reach the targets either increases or decreases.

The preliminary results suggest performing further investigations aimed at verifying whether more severe impaired subjects can be characterized by a more marked improvement of the upper limb impairment: further research will be performed to provide a validation of such method on a larger dataset.

\section{Conclusions}

The paper presented an innovative assessment method based on biomechanical parameters computed on force data recorded during the robot-aided upper limb rehabilitation in post-stroke subjects. The results demonstrated the hypothesis that, in such subjects, the 
motor recovery of planar reaching movements is based on the preservation of biomechanical and physiological properties.

Moreover, the proposed assessment method of an upper limb robot-aided rehabilitation has a high potential impact on the clinical decision process, as it can support the medical staff for identifying the most suitable rehabilitation approach for each post-stroke patient.

\section{Acknowledgements}

This study was partly supported by Fondazione Cassa di Risparmio di Volterra, Italy.

\section{References}

[1] S. Barreca, S.L. Wolf, S. Fasoli and R. Bohannon, Treatment interventions for the paretic upper limb of stroke survivors: a critical review, Neurorehab Neural Repair 17 (2003), 220-226.

[2] R.F. Beer, J.P. Dewald and W.Z. Rymer, Deficits in the coordination of multijoint arm movements in patients with hemiparesis: evidence for disturbed control of limb dynamics, Exp Brain Res 131(3) (2000), 305-319.

[3] R.W. Bohannon and M.D. Smith, Interrater reliability of a modified Ashworth scale of muscle spasticity, Phys Ther $\mathbf{6 7}$ (1987), 206-207.

[4] J.H. Carr and R.B. Shepherd, A motor learning model for rehabilitation. in: J.H. Carr and R.B. Shepherd eds, Movement Science, Foundations for Physical Therapy in Rehabilitation, 2nd edn, Aspen Publishers, Gaithersburg, Maryland, USA, 2000, pp. 33-110

[5] J. Chae, D. Mascarenhas, D.T. Yu, A. Kirsteins, E.P. Elovic, S.R. Flanagan, R.L. Harvey, R.D. Zorowitz and Z.P. Fang, Poststroke shoulder pain: its relationship to motor impairment, activity limitation, and quality of life, Arch Phys Med Rehabil 88(3) (2007), 298-301.

[6] R. Colombo, I. Sterpi, A. Mazzone, C. Del Conte, G. Minuto and F. Pisano, Measuring changes of movement dynamics during robot-aided neurorehabilitation of stroke patients, IEEE Trans Neural Syst Rehabil Eng 10(1) (2010), 75-85.

[7] W.W. Dawnie, P.A. Leatham, V.M. Rhind, V. Wright, J. Branco and J.A. Anderson, Studies with pain rating scales, Ann Rheum Dis 37 (1978), 378-381.

[8] S.E. Fasoli, H.I. Krebs, J. Stein, W.R. Frontera, R. Hughes and N. Hogan, Robotic therapy for chronic motor impairments after stroke: follow-up results, Arch Phys Med Rehabil 85(7) (2004), 1106-1111.

[9] M. Ferraro, J.H. Demaio, J. Krol, C. Trudell, K. Rannekleiv, L. Edelstein, P. Christos, M. Aisen, J. England, S. Fasoli, H.I. Krebs, N. Hogan and B.T. Volpe, Assessing the motor status score: a scale for the evaluation of upper limb motor outcomes in patients after stroke, Neurorehab Neural Re 16(3) (2002) 283-289.
[10] M. Ferraro, J.J. Palazzolo, J. Krol, H.I. Krebs, N. Hogan and B.T. Volpe, Robot-aided sensorimotor arm training improves outcome in patients with chronic stroke, Neurology 61(11) (2003), 1604-1607.

[11] C. Gowland, P. Stratford, M. Ward, J. Moreland W. Torresin, S. Van Hullenaar, J. Sanford, S. Barreca, B. Vanspall and N. Plews, Measuring physical impairment and disability with the Chedoke-McMaster Stroke Assessment, Stroke $\mathbf{2 4}$ (1993), 58-63.

[12] C. Gowland, S. Van Hullenaar, W. Torrensin, J. Moreland, B. Vanspall, S. Barreca, et al., Chedoke-McMaster stroke assessment: development, validation and administration manual, Chedoke-McMaster Hospitals and McMaster University, Hamilton, Ontario, 1995.

[13] J.W. Krakauer, Motor learning: its relevance to stroke recovery and neurorehabilitation, Curr Opin Neurol 19(1) (2006), 84-90.

[14] H.I. Krebs, N. Hogan, M.L. Aisen and B.T. Volpe, Robotaided neurorehabilitation, IEEE Trans Rehabil Eng 6(1) (1998), 75-87.

[15] H.I. Krebs, B.T. Volpe, M.L. Aisen and N. Hogan, Increasing productivity and quality of care: robot-aided neuro-rehabilitation, J Rehabil Res Dev 37(6) (2000) 639-652.

[16] H.I. Krebs, M. Ferraro, S.P. Buerger, M.J. Newbery, A. Makiyama, M. Sandmann, D. Lynch, B.T. Volpe and N. Hogan, Rehabilitation robotics: pilot trial of a spatial extension for MIT Manus, J Neuroengineering Rehabil 1(1) (2004), 5 .

[17] G. Kwakkel, B.J. Kollen and H.I. Krebs, Effects of robotassisted therapy on upper limb recovery after stroke: a systematic review, Neurorehabil Neural Repair 22(2) (2008), $111-121$.

[18] P.S. Lum, C.G. Burgar, D.E. Kenney and H.F.M. Van der Loos, Quantification of force abnormalities during passive and active-assisted upper-limb reaching movements in poststroke hemiparesis, IEEE Trans Biomed Eng 6(46) (1999), 652-662.

[19] P.S. Lum, C.G. Burgar, P.C. Shor, M. Majmundar and H.F.M. Van der Loos, Robot-assisted movement training compared with conventional therapy techniques for the rehabilitation of upper-limb motor function after stroke, Arch Phys Med Rehabil 83(7) (2002), 952-959.

[20] P.S. Lum, C.G. Burgar and P.C. Shor, Evidence for improved muscle activation patterns after retraining of reaching movements with the MIME robotic system in subjects with post-stroke hemiparesis, IEEE Trans Neural Syst Rehabil Eng 12(2) (2004), 186-194.

[21] C. Orizio, R. Perini, B. Diemont, M.M. Figini and A. Veicsteinas, Spectral analysis of muscular sound during isometric contraction of biceps brachii, J Appl Physiol 68(2) (1990), 508-512.

[22] F. Posteraro, S. Mazzoleni, S. Aliboni, B. Cesqui, A. Battaglia, P. Dario and S. Micera, Robot-mediated therapy for paretic upper limb of chronic patients following neurological injury, J Rehabil Med 41(12) (2009), 976-980.

[23] G.B. Prange, M.J. Jannink, C.G. Groothuis-Oudshoorn, H.J. Hermens and M.J. Ijzerman, Systematic review of the effect of robot-aided therapy on recovery of the hemiparetic arm after stroke, J Rehabil Res Dev 43(2) (2006), 171184.

[24] D.J. Reinkensmeyer, L.E. Kahn, M. Averbuch, A. McKennaCole, B.D. Schmit and W.Z. Rymer, Understanding and treating arm movement impairment after chronic brain injury: 
progress with the ARM guide, J Rehabil Res Dev 37(6) (2000), 653-662.

[25] D.J. Reinkensmeyer, J.L. Emken and S.C. Cramer, Robotics, motor learning, and neurologic recovery, Annu Rev Biomed Eng 6 (2004), 497-525.

[26] R. Riener, T. Nef and G. Colombo, Robot-aided neurorehabilitation of the upper limb, Med Biol Eng Comput 43 (2005), $2-10$.

[27] S.H. Scott, The role of primary motor cortex in goal-directed movements: insights from neurophysiological studies on nonhuman primates, Curr Opin Neurobiol 13(6) (2003), 671-677.
[28] R.B. Shepherd, Exercise and training to optimize functional motor performance in stroke: driving neural reorganization? Neural Plast 8(1-2) (2001), 121-129.

[29] A. Shumway-Cook and M.H. Woolacott, Motor control: theory and practical application, 2nd edn, Lippincot, Philadelphia Williams \& Wilkins, eds., 2001.

[30] J. Stein, H.I. Krebs, W.R. Frontera, S.E. Fasoli, R. Hughes and N. Hogan, Comparison of two techniques of robot-aided upper limb exercise training after stroke, Am J Phys Med Rehabil 83(9) (2004), 720-728. 

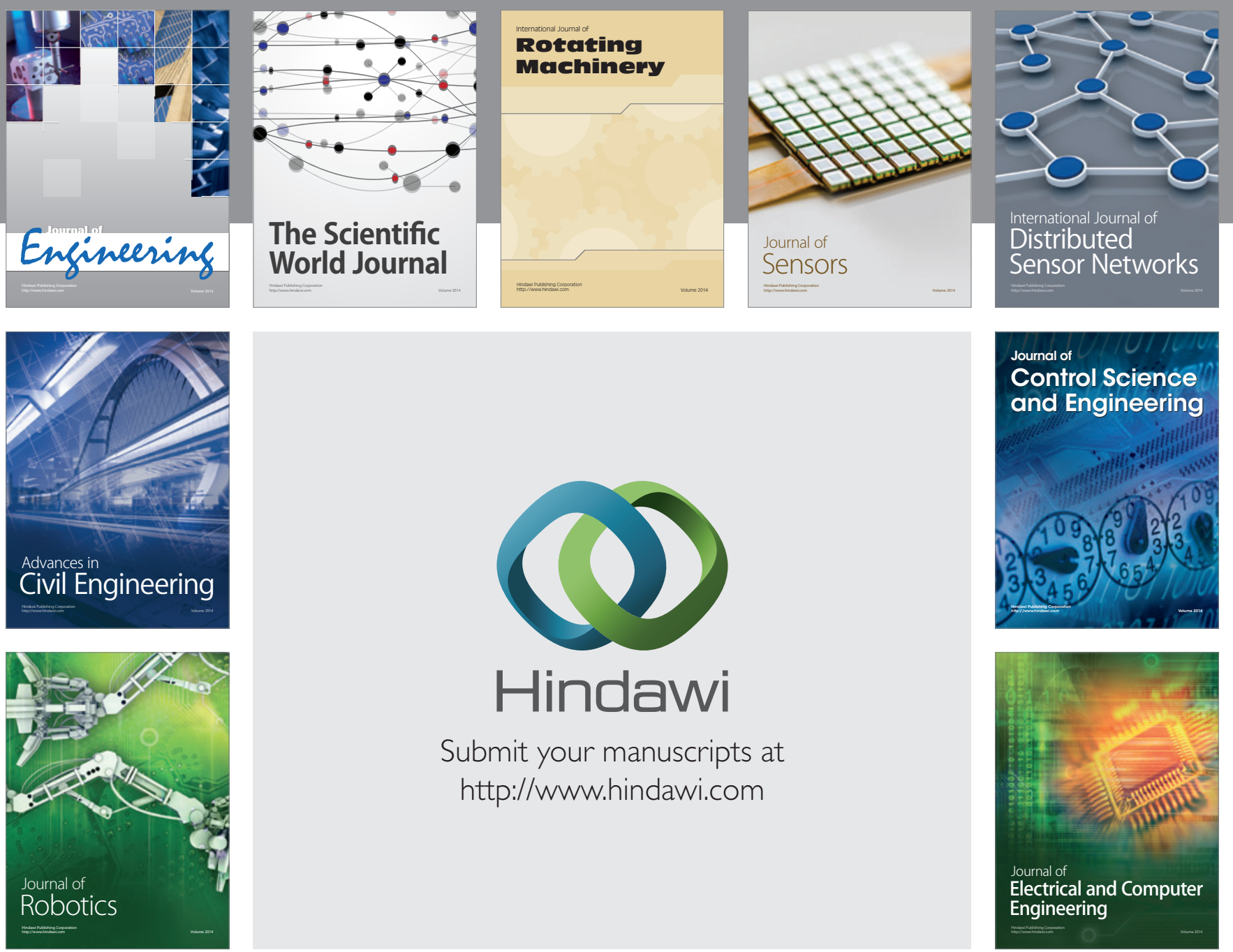

Submit your manuscripts at

http://www.hindawi.com
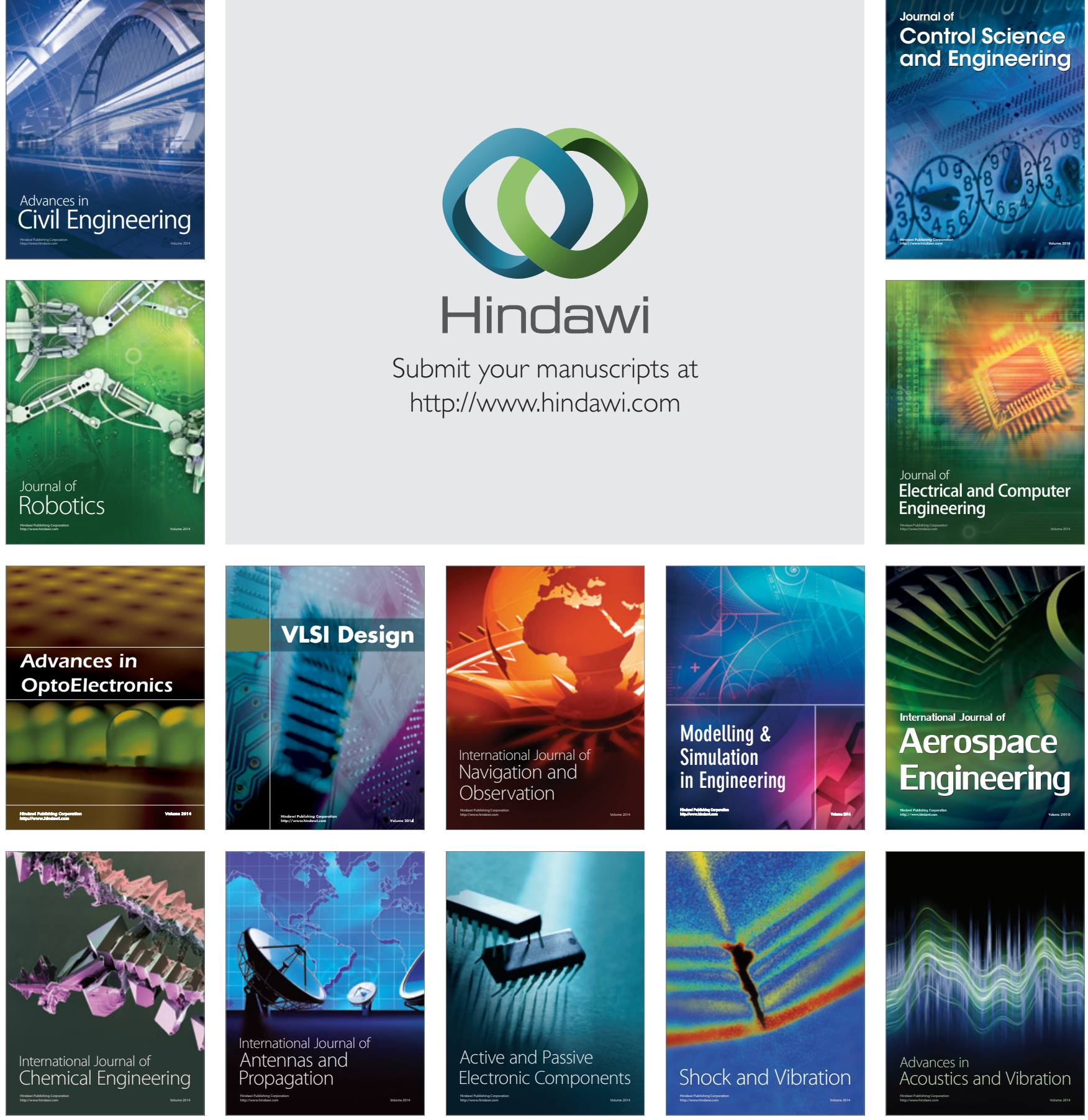